\title{
DEMOSTRANDO A PRIORI: MATEMÁTICAS Y REALISMO EN EL MYSTERIUM COSMOGRAPHICUM DE JOHANNES KEPLER
}

\author{
DEMONSTRATING A PRIORI: MATHEMATICS \\ AND REALISM IN JOHANNES KEPLER'S \\ MYSTERIUM COSMOGRAPHICUM
}

\author{
Francisco Javier LUNA" \\ Universidad de Barcelona
}

\begin{abstract}
Resumen: En su libro Mysterium Cosmographicum, Johannes Kepler postuló dos cuestiones estrechamente vinculadas: la existencia de un patrón de organización del espacio que da razón de la proporción entre las distancias planetarias, y que dichas distancias con respecto al cuerpo físico del Sol se vinculan con los periodos de cada planeta. Debido a lo anterior, Kepler creía que se podía demostrar a priori la correcta ordenación de los cielos, incluido su número y distancia, y en consecuencia demostrar finalmente la hipótesis de Copérnico. El presente artículo busca comprender la importancia que Kepler le daba a esa prueba a priori, así como el papel que jugó para su programa de investigación astronómica.
\end{abstract}

Palabras clave: Johannes Kepler, demostración a priori, sólidos platónicos, astronomía pitagórica.

ABSTRACT: In his book Mysterium Cosmographicum, Johannes Kepler proposed two closely related issues: first, that there is a pattern of organizing space that accounts for the ratio of planetary distances; secondly, that these distances, measured from the physical body of the sun, are related to the periods of each planet. In light of this, Kepler

* Investigador Posdoctoral en la UB. Montalegre, 6. E-08001 Barcelona.

E-mail: lechuza@unam.mx 
thought he could prove a priori the correct order of the heavens, including their number and distances, and consequently prove Copernicus' hypothesis. this article aims to understand the importance that Kepler gave to the a priori proof, and its role within his astronomical research program.

KeY wORDs: Johannes Kepler, a priori demonstration, Platonic solids, Pythagorean astronomy.

\section{Introducción}

Desde su formación universitaria en Tübingen bajo la tutela de Michael Mästlin, Johannes Kepler se convenció de que la teoría de Copérnico representaba la más auténtica descripción de los cielos que existía. ${ }^{1}$ Testimonio de este juvenil entusiasmo es el fragmento de un debate universitario: Orationis de motu terrae (ca. 1593), ${ }^{2}$ donde el joven Kepler defiende el movimiento de la Tierra y la centralidad del Sol "con argumentos físicos o, mejor, metafísicos". ${ }^{3}$ Este acercamiento al copernicanismo fue en principio más filosófico o incluso teológico que astronómico y la defensa del heliocentrismo por Kepler es en buena medida retórica, ofreciendo argumentos lógicos y elaborando un elogio al Sol muy parecido al que aparece en el De Revolutionibus de Copérnico. ${ }^{4}$ Así, Kepler escribe:

\footnotetext{
${ }^{1}$ La visión sobre Copérnico de Kepler como un cosmólogo innovador es en cierta forma atípica, pues Copérnico era tenido en el siglo XVI por un gran matemático pero su heliocentrismo, como teoría física, casi no se tomaba en cuenta, como explica Westman: "Indeed, certain parts of the new theory were to be adopted and preferred as consistent with the foundations of astronomy if interpreted in a framework where the earth was at rest, while other aspects were rejected or ignored as irrelevant or as possessing low truth content." (Westman, 1975, 167).

${ }^{2}$ Kepler, "Fragmentum Orationis de Motu Terrae", en Gesammelte Werke, vol. XX.1, C. H. Beck, Munich, 1938, pp. 147-149 [en adelante citada como $K G W$ ]. Nicholas Jardine hace un estudio de la importancia de esa obra tan temprana y también da una traducción al inglés: Nicholas Jardine, "Kepler's 'lightly woven cosmography': On the sources, identity and signifcance of Kepler-Ms 18, 238v-241r, Russian Academy of Sciences, St Petersburg" (NoirotOrdine, 2012, 453-465).

${ }^{3}$ Kepler, Mysterium cosmographicum, "Praefatio ad lectorem" [en adelante citado como $M C]$. Utilizo la versión en español de Eloy Rada: El secreto del Universo, Alianza, Madrid, 1992, y también la latina editada por Max Caspar: $K G W$, I, pp. 1-80 y la segunda edición $K G W$, VIII, pp. 7-128

${ }^{4}$ Nicolás Copérnico, Sobre las revoluciones de los orbes celestes, I, 10 (1989, 35).
} 
To start with, not even a blind man would deny to you that of all the bodies there are in the World the most excellent is the Sun, whose entire essence is nothing but the purest light, than which no star is greater [major], which is the one and only producer, preserver and cherisher of all things, the fountain of light, the wellspring of abundant heat, most beautiful, most clear and pure to the sight, the origin of vision, the revealer of all colours, itself lacking colour; called the king of the planets for its motion, the heart of the World for its power, the eye for its beauty; and which all of us would regard as the one worthy thing which the best and greatest God would inhabit with the blessed angels, if He should please to have a corporeal home and could be contained in a place. ${ }^{5}$

Esta exaltada visión tiene además un claro componente teológico, pues como se dice al final de la cita, el Sol mismo podría verse como la sede terrenal del poder divino. James Voelkel no duda en afirmar que ya desde entonces Kepler hacía una analogía entre Dios Padre y el Sol: "The sun, the most resplendent body, was situated in the center, whence it distributed light, heat, and motion to the planets. It represented God the Father". ${ }^{6}$ Kepler contempla el ordo planetarium como una estructura armónica que refleja la perfección de la mente divina. El peso que le da al centro, idea seminal de su programa de investigación y representación de Dios padre, se asocia perfectamente con una visión heliocéntrica del Mundo. Pero no debemos olvidar que Kepler contempla el conjunto entero como una gran armonía, aunque el centro sea el eje, cada sección debe mantener igualmente vínculos armónicos con todas las demás, deben existir proporciones perfectas del centro con las partes y entre las partes mismas.

\footnotetext{
${ }^{5}$ Noirot-Ordine, 2012, 463. "Para empezar, ni siquiera un ciego podría negar que de todos los cuerpos que hay en el mundo, el más excelente es el Sol, cuya esencia total no es sino la luz más pura, mayor que ninguna estrella. El uno y único productor, conservador y animador, fuente de la luz, fuente del calor abundante, la más bella, la más clara y pura a la vista, el origen de la visión, el revelador de todos los colores, que carece de color; rey de los planetas por su movimiento, el corazón del mundo por su poder, el ojo por su belleza. Y que todos nosotros consideraríamos el único digno, para que Dios Opt. Max. habitaría con los ángeles benditos, si poseyera un domicilio corpóreo y pudiera estar contenido en un lugar". Las traducciones son mías salvo que se indique otra cosa.

${ }^{6}$ Voelkel, 1999, 20. "El sol, el cuerpo más resplandeciente, fue situado en el centro, desde donde distribuía luz, calor y movimiento a los planetas. Éste representaba a Dios padre”.
} 
Ahora bien, calificar esta visión sólo de teológica y poética sería un error, pues aunque no se adentra demasiado en los problemas geométricos que conlleva, contiene ya un giro novedoso al adoptar una postura realista, es decir, se toma la cosmología de Copérnico como una descripción física de los cielos. Gracias a esa idea, Kepler trasciende al mero encomio y comienza a apuntar hacia algunas consecuencias físicas de la teoría, fascinado con las opciones que abría la nueva ordenación de los cielos para la especulación sobre las causas de los fenómenos astronómicos. ${ }^{7}$ De esta forma se abre la puerta para buscar una proporción entre el centro y los periodos planetarios, pero también se buscará una proporción entre los propios planetas, su número y distancias. Todo ello bajo una visión muy pitagórica de la mente divina, por lo que en última instancia lo que se intenta es descubrir el plano arquitectónico del Mundo.

\section{Del problema de la fuerza solar al misterio geométrico}

El cambio más importante en su comprensión de Kepler del heliocentrismo fue el claro papel que asigna al Sol como "primum movens" , dando con ello un nuevo significado a su posición en el centro del Mundo. Esta idea no era sólo metafísica, sino que dará pie a un tratamiento matemático del problema para relacionar las distancias individuales de cada planeta al Sol con sus velocidades particulares: "as from the centre and heart of the World, it should extend itself

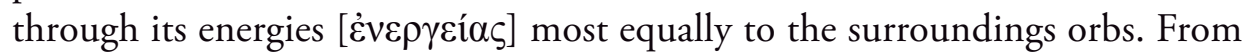
this, consequently, the proportion of the speeds of the planets can easy be estimated [...] impinging on them with equal force and quickening the revolutions of each in proportion to the proportions of their circuits [pro ratione ambitus]".? Desde estas líneas tempranas se marca la empresa de vincular la fuerza motriz del

\footnotetext{
${ }^{7}$ Como bien señala Alexandre Koyré $(1973,129)$ : “The part it [the Sun] plays in the Ptolemaic Universe is just as important, even more perhaps, that in the Copernican, as Kepler showed [in the Mysterium] [...] There is this difference, however; with Ptolemy, this preeminent rôle of the Sun is based on nothing, whilst with Copernicus it forms part of the very structure of the system, which replaces the empirical disorder of this predecessor by a rational arrangement”.

${ }^{8} K G W$, XX.1, 148. Véase también el análisis del Fragmentum Orationis de motu terrae que hace James R. Voelkel (2001, 28 y 29).

${ }^{9}$ Noirot-Ordine, 2012, 464. "porque desde el centro y corazón del Mundo, debe extenderse a través de sus energías más uniformemente hacia los orbes al rededor. Desde donde, en consecuencia, la razón de las velocidades de los planetas puede con facilidad ser estimada [...] repartiendo sobre de ellos con igual fuerza y velocidad las revoluciones de cada uno en proporción con sus circuitos".
} 
Sol con la proporción entre distancia desde el centrum et cor mundi y la velocidad del periodo. Así, la propuesta de Kepler de asignar un papel físico y mensurable a las fuerzas que actúan sobre los cuerpos celestes transformó argumentos anteriormente especulativos a hipótesis físicas por medio de funciones matemáticas que deben comprobarse en los hechos. La idea de una fuerza motriz singular del Sol guiará en muchos sentidos la investigación astronómica posterior de Kepler y su fructífero intento de unir la filosofía de la naturaleza y la astronomía. Evidentemente, esa idea se basa por completo en la premisa de que la hipótesis heliocéntrica es verdadera y que, así como existe una razón para que el Sol sea el centro físico del sistema, existen razones suficientes para la completa revisión del 'ordo planetarum' llevada a cabo por Copérnico.

Ya como astrónomo profesional, Kepler intentó descubrir la razón concreta entre la velocidad de cada astro y su distancia al Sol, que, como ya había anotado en su De motu terrae, es de forma general y según el modelo de Copérnico inversamente proporcional. Esta búsqueda de una razón matemática encaminó a Kepler a indagar sobre la existencia de algún patrón en los cielos que explicara las proporciones entre las distancias calculadas por Copérnico, ${ }^{10}$ la cual en caso de ser descubierta demostraría a su vez que la teoría copernicana era verdad. El buscar vínculos armónicos con números no era en sí mismo un tema novedoso, relacionar las distintas partes del cielo eran un antigua empresa de la astrología/ astronomía, aunque la búsqueda habitualmente se hacía más bien a nivel simbólico. Los astrólogos solían buscar patrones que relacionaran los astros entre sí, muchas veces a través de complicadas analogías. Para ese tipo de búsquedas, "las ventajas del orden matemático de Copérnico sobre Ptolomeo"11 estribaban precisamente en que al colocar al Sol como centro del sistema se ponía de manifiesto de inmediato la relación armónica entre el astro rey y la velocidad planetaria. Pero Kepler sostuvo que la posición del Sol en el sistema obedecía a una razón física y no sólo geométrica o simbólica, idea que le condujo a reforzar su convicción de que existía una fuerza única en el cuerpo solar que era la causante del movimiento de todos los planetas y también a afirmar que que dicha fuerza se debilitaba, igual que la luz, con la distancia. La hipótesis de la fuerza solar socavaba la tradicional explicación de que las esferas sólidas transportaban a los planetas inmóviles "sicut nodi in tabula" o la concepción animista de que los planetas se

\footnotetext{
${ }^{10}$ Van Helden, 1985, 44.

${ }^{11}$ Kepler, $M C$, "Praefatio ad lectorem" $(1992,66)$. Existieron partidarios de Copérnico cuyo interés estaba precisamente en la armonía astrológica y en la exaltación solar de corte hermético, por ejemplo Pontus de Tyard. Heller, 1996, 14-16.
} 
movían por sus propias inteligencias internas y particulares "sicut pisces in mare et aves in aëre". ${ }^{12}$ Según la concepción kepleriana, debía ser posible determinar matemáticamente la razón entre las distancias y las diversas velocidades, pues ambas cuestiones estaban conectadas con la misma fuerza física que se extendía desde el centro del sistema.

Pese a la aparente simplicidad de ese planteamiento, ya en la práctica el descubrimiento de la razón exacta entre distancia y velocidad no fue en absoluto sencillo. Kepler relata en el Mysterium que buscó alguna función matemática que encajara entre las distancias ofrecidas por Copérnico y la velocidad de cada astro, "porque si Dios (pensaba yo) ha dotado a los orbes de un movimiento en correspondencia con sus distancias, también habría acomodado las propias distancias a alguna otra cosa". ${ }^{13}$ Armado al principio con datos de las Tablas Pruténicas y del De Revolutionibus sobre distancias y periodos, ni siquiera le fue posible a Kepler encontrar una razón entre distancia y periodo cuya relación parecía evidente, para no decir sobre encontrar el patrón de distancias o, mucho menos, la forma de acción concreta de la fuerza solar. El Mysterium narra que incluso intentó intercalar planetas fantasmas entre Júpiter y Marte y entre Venus y Mercurio para completar una posible escala geométrica o aritmética. Otro intento igualmente fracasado fue el uso de funciones trigonométricas, específicamente "si acaso la distancia de algún planeta dentro del mismo cuadrante no sería como el resto del seno, mientras su movimiento sería como el resto del seno del complemento [o coseno]" ${ }^{14}$ Pese a no conseguir su objetivo, Bruce Stephenson resaltó particularmente la importancia de esta temprana especulación y le dio un desarrollo en notación actual:

That is, if we introduce the parameter $\Theta[\ldots]$ he speculated that the ratio of speed to distance was $(1-\cos \Theta) /(1-\sin \Theta)$, with a value of $\Theta$ particular to each planet. This theory, if we may so term it, had most of the same flaws as the other one (notably a lack of empirical adequacy), but at least it did

\footnotetext{
${ }^{12}$ Granada, 2010, 111-141. Punto importante de ese debate fue la crítica de Tycho Brahe en De mundi aetheri recentioribus phaenomenis, cap. 8 (1588) y en su correspondencia con Rothmann, editada en 1696, donde había argumentado contra los orbes sólidos y había optado por una concepción de cielos líquidos justificados con cierta base bíblica. Al respecto véase Blair, 1990, 355-377.

${ }^{13}$ Kepler, $M C$, "Praefatio ad lectorem" (1992, 67). El subrayado es mío.

${ }^{14}$ Ibid.
} 
predict that the fixed stars would be motionless at the (finite) distance corresponding to $\Theta=0$, as Kepler believed. It further predicted that the force of motion would be maximal, perhaps infinite, at the centre where was the sun, representing motion in its very essence. $(1987,9)^{15}$

Este primer modelo kepleriano entre el periodo y la distancia condujo a un callejón sin salida, pero puso sobre la mesa un problema central para el nuevo modelo. Kepler no tenía la menor idea de qué patrón de medida usar y su estrategia para descubrirlo se basaba únicamente en el proceso de prueba y error. La búsqueda dio un giro brusco a raíz de una observación casual, la idea de usar las formas geométricas para la razón de las distancias entre los planetas, la cual le vino al germano al notar una consecuencia cosmológica del heliocentrismo: la existencia de espacios intermedios entre las tradicionales esferas celestes. ${ }^{16}$ Como explican Goldstein y Hon:

In fact, Kepler was the first to draw the Ptolemaic and Copernican models to scale and he noticed that for Copernicus there were significant gaps between adjacent planetary orbs, that is, the minimum distance of a planet from the Sun was not equal to the maximum distance of the planet closer to the Sun where the order is: Mercury, Venus, Earth, Mars, Jupiter, and Saturn. $(2005,74-111)^{17}$

\footnotetext{
15 "Es decir, si introducimos el parámetro $\Theta[. .$.$] él especula que la relación de velocidad a$ distancia era $(1-\cos \Theta) /(1-\sin \Theta)$, con un valor de $\Theta$ particular para cada planeta. Esta teoría, si así la llamamos, tenía la mayoría de los mismos defectos que la otra (notablemente falta de adecuación empírica), pero al menos predijo que las estrellas fijas estarían inmóviles a la distancia (finita) correspondiente a $\Theta=0$, como creía Kepler. Predijo además que la fuerza del movimiento sería máxima, tal vez infinita, en el centro donde estaba el sol, representando el movimiento en su esencia misma”.

${ }^{16}$ Aunque Kepler fue criticado por Brahe por hablar de esferas, la idea de orbe en Kepler es más bien simbólica y se usa para referir el espacio comprendido entre el perihelio y el afelio de cada planeta.

17 "Kepler fue el primero en dibujar los modelos ptolemaico y copernicano a escala y se dio cuenta de que para Copérnico había huecos significativos entre orbes planetarios adyacentes, es decir, la distancia mínima entre un planeta y el Sol no era igual a la distancia máxima de el planeta anterior más cercano al Sol, donde el orden es: Mercurio, Venus, Tierra, Marte, Júpiter y Saturno".
} 
En el trascurso de sus disquisiciones, Kepler reflexionó sobre esas distancias entre los orbes planetarios que Copérnico había afirmado que eran proporcionales y no plenos como en la teoría planetaria de Ptolomeo. El cambio de perspectiva fue una consecuencia del movimiento de traslación de la Tierra en el heliocentrismo, el cual explicaba de una nueva forma la llamada "segunda anomalía" o movimiento de retrogradación de los planetas superiores; desde el geocentrismo dicha anomalía sólo se podía explicar haciendo uso de enormes epiciclos para dar cuenta del fenómeno; sin embargo, desde el heliocentrismo, la segunda anomalía no era un movimiento real sino un efecto óptico causado por el movimiento anual de la Tierra. En consecuencia, Copérnico eliminó los grandes epiciclos, cambiándolos por unos pequeños sólo para ajustar la velocidad; el proceso dejó una serie de espacios vacíos entre los caminos de los planetas, los mismos que Copérnico afirmó estaban regidos por proporciones. Sin embargo, dicho argumento no era del todo satisfactorio pues no explicaba realmente la necesidad de dichas proporciones ni tampoco indaga cuáles podrían ser esas proporciones concretas, como explica James Voelkel:

It's not clear, however, that Copernicus's metaphysical principle of commensurability is any less idiosyncratic than Ptolemy's principle of plenitude. One might ask, as Kepler would, why were the planets located at precisely those distances and not any others? And why was there so much useless intervening space? But these were not issues Copernicus addressed. (2001, $21)^{18}$

Kepler narra en el Mysterium cómo se dio cuenta de que en los espacios vacíos se podían insertar figuras geométricas y así dar cuenta de las proporciones entre las esferas. Intentando primero con figuras planas, finalmente Kepler recuerda que sólo existen cinco sólidos regulares y asume que éstos eran las figuras que estaba buscando pues encajan entre los seis planetas de la teoría copernicana. A partir de ese punto, el Mysterium se centra en explicar ese secreto arcano de la

\footnotetext{
${ }^{18}$ De cualquier forma, no es claro que el principio metafísico de conmensurabilidad de Copérnico sea menos idiosincrático que el principio de plenitud de Ptolomeo. Uno podría preguntarse, como lo haría Kepler, ¿por qué los planetas estaban situados exactamente a esas distancias y no a otras? ¿Y por qué había tanto espacio inútil? Pero éstas no fueron cuestiones que Copérnico abordara.
} 
ordenación de los planetas y se deja de lado el problema de la fuerza solar misma que no aparecerá hasta los últimos capítulos. Así, en apariencia, Kepler ha relegado el problema físico en favor de una complicada especulación geométrica, pero en realidad desde un principio el objetivo era explicar no sólo la relación entre distancia y periodo, sino también la proporción entre las distancias. En este sentido, el Mysterium fue más bien una cambio de enfoque. En lugar de intentar cuantificar la fuerza física por su efecto (el movimiento) y así determinar las proporciones de las distancias, se empezó desde el supuesto descubrimiento de la razón misma entre distancias y, creyendo tener ese dato seguro, el objetivo era regresar luego a la fuerza física. Recordemos que Kepler estaba en la búsqueda no sólo de un razón que uniera distancia con periodo, sino de un patrón más amplio, una verdadera gran armonía, que explicara el número exacto de planetas, su distribución real por el espacio e incluso el grosor de sus órbitas.

El problema de la proporción entre distancias estaba ya presente desde el $D e$ motu terrae, ahí se señala que el movimiento puede dividirse en una infinidad de grados [motus infinitis prope partibus], lo cual parecía excluir la posibilidad de explicar el número de cuerpos del sistema y dejaba, desde la perspectiva de Kepler, incompleta cualquier explicación. Esa ańoranza por encontrar una gran armonía, nos ayuda a comprender la emoción y aprecio de Kepler a su hipótesis geométrica, pues no sólo le aportaba un patrón de medida entre radio de la órbita y su circunferencia, sino también creía que explicaba el grosor entre el perihelio y el afelio, e incluso pensaba que podía servirle para corregir los datos computados de las distancias. La contrapartida de esa grandiosa construcción apriorística era que existía una forma de comprobarla, los sólidos debían encajar de forma perfecta en los espacios intermedios entre los seis planetas según las distancias reales, ${ }^{19}$ justificando en el proceso que sólo existiera ese número exacto de planetas.

El resultado de esa investigación fue el primer libro sobre los cielos del joven Kepler, su obra Prodromus Dissertationum Cosmographicarum continens Mysterium cosmographicum (Precursor de consideraciones cosmográficas conteniendo el misterio de la cosmografía, 1596), donde se afirma triunfalmente que los cinco sólidos platónicos son la base de la organización del espacio. La elección de lo sólidos platónicos conllevaba un profundo simbolismo, estos sólidos son un conjunto único de figuras regulares estudiadas en el libro XIII de los Elementos de Euclides, muy valoradas desde los pitagóricos griegos hasta el Renacimiento pues

${ }^{19}$ El trabajo de calcular los espacios fue sobretodo obra de Mästlin, como bien lo argumentó, sobre todo desde el tercer diagrama del MC, Grasshoff $(2012,57-74)$. 
fueron tenidas como símbolos de perfección. Así, por ejemplo, para el platónico Proclo los poliedros regulares representan la culminación de la geometría griega, como afirma en su Comentario al primer libro de los Elementos de Euclides. Proclo incluso creía que la meta final de la obra de Euclides era fundamentar de manera inobjetable las propiedades geométricas de los cinco sólidos regulares que Platón había usado en su Timeo, y en el Comentario asegura que "Euclid belonged to the persuasion of Plato and was at home in this philosophy; and this is why he thought the goal of the Elements as a whole to be the construction of the so-called Platonic figures". ${ }^{20}$

La interpretación teologizante de Proclo de la geometría, con todo su simbolismo armónico y místico, gozaba de gran estima en el ambiente académico de las universidades reformadas donde se formó Kepler como aspirante a teólogo. Estas ideas tendían un puente entre la visión de la naturaleza como obra divina y el paradigma de la demostración matemática como aspiración del espíritu humano. Según afirma Charlotte Methuen, el propio Philipp Melanchthon, arquitecto de la educación universitaria reformada, tuvo en alta consideración el libro de Proclo:

Proclus too emphasises the usefulness of linking mathematical demonstration and the divinity of mathematics in his commentary to the first book of Euclid's Elements, and although his approach has a generally Platonist orientation, his understanding of demonstration is fundamentally Aristotelian. His interpretation may also have been attractive to Melanchthon, who almost certainly knew of Proclus' commentary since it had been edited by Simon Grynaeus [...] [but] Melanchthon's praise of the mathematics is not praise of the ability of the mathematics to describe an actual situation. It is,

\footnotetext{
${ }^{20}$ Proclus, 1970, $\$$ 68, 57. "Euclides pertenecía a la enseñanza de Platón y se sentía en casa con su filosofía; Y por eso pensó que el objetivo de los Elementos como un todo era la construcción de las llamadas figuras platónicas". Para Proclo, Pitágoras "it was who discovered the doctrine of proportionals and the structure of the cosmic figures." ( $\$ 66$, p. 53$)$, por lo cual Euclides seguía la tradición del pitagorismo antiguo. Kepler comparte esta visión y no duda en hacer también a los copernicanos herederos de la filosofía pitagórica, por citar un ejemplo en la carta a Mästlin de junio de 1589: "Pythagoras aller Copernicaner großvatter." $K G W 13$, Nr. 99, 219.
} 
however, perhaps overstating the case to characterise Melanchthon's praise of mathematics as a rhetorical device. $(1998,97)^{21}$

Esa visión de la matemática como un aspecto divino se refleja en la obra de Kepler, quien coincide con Melanchthon en la visión de un Dios geómetra que realiza sus obras con suma perfección. Sin embargo, mientras que para Melanchthon la matemática es sólo una propedéutica del teólogo y sus demostraciones un ideal hermenéutico, para Kepler la idea de un Dios geómetra es más que una metáfora. Para nuestro astrónomo, el "libro de la naturaleza" es una realidad que se despliega efectivamente delante de los ojos del maravillado espectador que es capaz de leer los caracteres matemáticos en que está escrito. En la dedicatoria del Mysterium escribe Kepler:

Pero aquí está aquel libro de la naturaleza, tan celebrado en los discursos sagrados, propuesto por Saulo a los gentiles en el cual se contempla a Dios como al Sol en el espejo de agua. ¿Por qué, pues, los cristianos nos deleitaremos menos en esta contemplación, siendo nuestro cometido celebrar a Dios con verdadero culto, venerarlo y admirarlo? Esto se hace con ánimo tanto más devoto cuando entendemos más correctamente cuáles y cuántas cosas ha fundado nuestro Dios. $(1992,55)$

Kepler lleva a cabo así una inversión del ideal de Melanchthon, pues no es la matemática la que debe inspirar con su rigor la retórica del teólogo, más bien es la matemática en sí misma la clave de la interpretación de la obra divina, lo

\footnotetext{
21 "Proclo también enfatiza la utilidad de vincular la demostración matemática y la divinidad de las matemáticas en su Comentario al primer libro de los Elementos de Euclides, y aunque su enfoque tiene una orientación generalmente platónica, su comprensión de la demostración es fundamentalmente aristotélica. Su interpretación también pudo haber sido atractiva para Melanchthon, quien casi seguramente sabía del comentario de Proclo desde que había sido editado por Simon Grynaeus [...] [pero] el elogio de Melanchthon a las matemáticas no es elogio de la capacidad de las matemáticas para describir un situación real. Es, no obstante, quizás exagerar el caso de caracterizar los elogios de Melanchthon de las matemáticas como un dispositivo retórico".
} 
cual conlleva elevar el ideal científico a tarea teológica. ${ }^{22}$ En base a este ideal de una matemática divina inscrita en el mundo físico, se llega a la conclusión de que para comprender la estructura de los fenómenos naturales e incluso poder predecir sus efectos basta con descubrir el patrón matemático que gobierna a los entes. En el caso concreto de la astronomía, descubrir el auténtico patrón de la estructura del cosmos significaría que se podría demostrar a priori la correcta ordenación de los cielos y, por consecuencia, demostrar de forma definitiva cuál de los modelos astronómicos en conflicto era verdadero. Kepler creía haber descubierto el arcano del cosmos en los sólidos regulares y gracias a ello estaba seguro de poder demostrar a priori la hipótesis copernicana. ${ }^{23}$

Debido a esa convicción, el Mysterium de Kepler distaba mucho de las obras astronómicas en el sentido en que se definían éstas en aquella época. La astronomía tradicional fue ante todo una actividad práctica destinada a calcular las posiciones planetarias para corregir el calendario, así como para usos navales y astrológicos. Para ello el astrónomo podía valerse de hipótesis de trabajo o modelos de los cielos sin comprometerse con la verdad de los mismos. Como ciencia, la astronomía guardaba un carácter más bien ambiguo y en general dominaba una cierta actitud escéptica en la comunidad de astrónomos sobre la posibilidad de acceder a la verdadera estructura del cosmos. ${ }^{24}$ En contraste con lo anterior, la obra de Copérnico y la de Kepler aspiraba a describir de forma auténtica la estructura de los cielos y las relaciones entre sus partes. Kepler tituló a su primer libro "Prodromus", indicando que era sólo la primera de una serie de obras que pretendía continuar, y añadiendo "dissertationum cosmographicarum" intentado señalar su convicción de estar ofreciendo una auténtica descripción del cosmos. Sin embargo el término causó no poca confusión, pues aunque la cosmografía era la disciplina con principios matemáticos más cercana a una filosofía de la naturaleza, el término en sí no lograba captar la unión de astronomía y física. ${ }^{25}$

\footnotetext{
22 Por ejemplo, Kepler llama a las astrónomos "sacerdotes dei altissimi ex parte libri Naturae" en la carta a Hans Georg Herwart del 26 de marzo de 1598. KGW, XIII, Nr. 91, 193.

${ }^{23} \mathrm{El}$ problema del ordenamiento y proporciones de los cielos se arrastraba desde la época de los griegos y era aún de gran importancia dentro de la astronomía de la época. Ésta fue una de las razones centrales de Copérnico para corregir a Ptolomeo según Voelkel: "Copernicus's critique of Ptolemy's way of assembling the planetary models into cosmological system was that it had no commensurability- that is, that this arrangement of the universe had no common measure." (2001, 21).

${ }^{24}$ Sobre este problema y la innovación que significó la obra de Kepler, véase: Jardine (1984, 225-257).

${ }^{25}$ La palabra "cosmografía” era usada para referirse a manuales prácticos que incluían una descripción somera del Mundo y después se concentraban en las partes de la superficie
} 
El calificativo más adecuado para el profundo realismo físico con que Kepler abordó su astronomía sería el posterior concepto de "physica coelestis", que él introduce en el título de su gran obra Astronomia Nova (1609).

\section{La prueba a priori y la construcción de una astronomía física}

Aun sin el concepto preciso que unificara su propuesta, el objetivo del Mysterium de servir como una demostración a priori de la hipótesis copernicana denota la intención de inaugurar una cosmología geométrica, en contraste con la astronomía computacional que se construía a partir de las observaciones, es decir, a posteriori. Por supuesto los astrónomos anteriores y contemporáneos a Kepler utilizaban alguna teoría física como guía para interpretar sus observaciones, específicamente la física de Aristóteles la cual se daba por sentado que era un saber prácticamente acabado, pero el hecho de que un astrónomo aportara sus propias teorías físicas y que las desarrollara a la par que sus modelos matemáticos eran algo único al punto de ser difícil de comprender por sus colegas, según explica Stephenson:

It is not physics but the use to which he [Kepler] put physics in his astronomy. Here Kepler went his own way [...], his other contemporaries simply did not understand what Kepler was trying to do. Even if we are not certain exactly how he was bending his physical vocabulary to his needs, we can follow his attack upon the essentially new problems of developing physical theory to accompany his developing astronomy. $(1987,4)^{26}$

terrestre, incluyendo además una serie de herramientas matemáticas prácticas. Por ejemplo, existían la Cosmographia (1524) de Pedro Apiano, ampliada por Gemma Frisius, que explicaba los movimientos de las estrellas, los instrumentales de la astronomía, las medidas que se ocupaban y el sistema de navegación por los astros; la Cosmographia in quatuor libros distributa summo ordine (1585) de Francesco Barozzi, donde se explicaban los principios matemáticos y geométricos de la astronomía, las propiedades de las figuras planas y sólidas, la esfera del mundo, los movimientos de los astros y las influencias mágicas de éstos; algo diferente era la Cosmographia universalis (1544) de Sebastian Münster, que, además de la parte dedicada a los movimientos celestes, se ocupaba de describir los países, las religiones y los gobiernos de Europa.

26 "No es la física sino el uso que él [Kepler] impuso la física en su astronomía. Aquí Kepler fue por su propio camino, [...] sus otros contemporáneos simplemente no entendieron lo que Kepler estaba tratando de hacer. Incluso si no estamos seguros de cómo estaba doblando su 
La innovadora postura realista y apriorística del Mysterium le trajeron fuertes críticas por parte de aquellos astrónomos que creían que dicha astronomía era imposible, incluido el futuro patrón de Kepler, Tycho Brahe ${ }^{27}$. Pero esas mismas características fueron las que ganaron el aprecio del maestro de Kepler, el también copernicano Michael Mästlin, quien apoyó decididamente la publicación del libro, editándolo, gestionando permisos, componiendo cálculos y diagramas. Tras conocer la idea de su alumno, Mästlin le escribe que: "Cada vez que pienso sobre tus demostraciones que doctísimamente prueban a priori las hipótesis de Copérnico, vehementemente me congratulo de que finalmente un docto varón se haya atrevido a romper el cerco de esa turba de imprudentes matemáticos que contradicen las hipótesis de Copérnico". ${ }^{28}$

El Mysterium cosmographicum es bastante claro en su realismo matemático y no duda en justificarlo con argumentos filosóficos y teológicos. El libro comienza afirmando que los cielos fueron trazados por el Optimus Maximus siguiendo un patrón geométrico preciso. Kepler escribe: "Es mi intención, lector, demostrar en este pequeño libro que el Creador Óptimo Máximo, al crear este mundo móvil y en la disposición de los cielos se atuvo a los cinco cuerpos regulares [...] y también que en función de su naturaleza ajustó su número, sus proporciones y la razón de sus movimientos" ${ }^{29}$ La postura epistémica del Mysterium sigue de cerca al Timeo platónico, afirmando una identidad entre las formas geométricas, que sirven como paradigmas, y las estructuras formales de los cielos físicos. La famosa cita del Timeo: "Pues ni ahora ni nunca se puede evitar que el mejor de

vocabulario físico a sus necesidades, podemos seguir su ataque hacia los problemas esencialmente nuevos de desarrollar una teoría física para acompañar su astronomía en desarrollo.”

27 "If astronomy must be restored a priori by determining the motions according to these geometrical bodies rather than building this restoration on accurate observations obtained a posteriori, as you imply, we will most certainly have to wait quite a long time, if no forever in vain” Tycho a Maestlin 21 Abril /1 mayo 1598, KGW, XIII, n. 94, 200 en Granada-BonerTessicini, 2016: 205. La crítica de Brahe no fue la única pero sí una de las más importantes, tanto por su prestigio como por la posterior relación de trabajo con Kepler. Brahe, si bien no era del todo opuesto a introducir consideraciones armónicas en la astronomía, se oponía a predecir resultados de postulados a priori, apoyando la visión de una astronomía que por necesidad debe ser a posteriori, a partir de las observaciones precisas como las que él mismo había reunido (Voelkel, 2001, 84 y 85).

${ }^{28}$ Carta de Mästlin a Kepler del 27 de febrero de 1596. "Quoties de tuis demonstrationibus, quibus Copernici hypotheses à priori doctissimè probas, cogito, vehementer mihi gratulor, quod tandem aliquis vir doctus inueniatur, qui isti imprudentium Mathematiculorum turbae, his Copernici hypothesibus obloquentium, ora obtundere audet.” $K G W, \mathrm{XIII}, \mathrm{Nr}$. 29, 54.

${ }^{29} M C$, "Praefatio ad lectorem" (1992, 65). 
los seres produzca la más bella de las obras", ${ }^{30}$ la retomó Kepler y con ella también reclamó los principios que él creía pertenecían a la cosmología pitagórica de

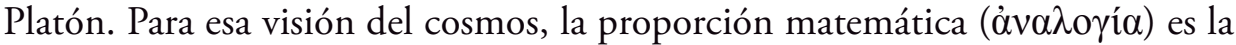
responsable de conducir los objetos físicos del desorden al orden y en consecuencia el matemático se convierte en el más calificado para hablar de "la naturaleza del universo".$^{31}$ Además de esa idea fundamental, Kepler también compartió con Platón la visión de un Mundo finito y único.

La profunda influencia del platonismo y del pitagorismo es patente en toda la obra kepleriana, aunque esto no le impidió marcar también cierta independencia y proclamar que las teorías de Platón "no necesariamente deban tomarse literalmente". ${ }^{32}$ Existen algunas diferencias entre el Timeo y la cosmología de Mysterium que merece la pena señalar para comprender la especificidad de la propuesta del germano. Para empezar, en el Timeo, la arquitectura matemática del cosmos es diferente a la del Mysterium, pues se desarrolla por medio de proporciones en dos series geométricas que marcan las divisiones de la esfera del Mundo, usando en cambio a los cuatro primeros poliedros regulares para la descripción de los cuatro elementos y dejando sólo al dodecaedro "para el universo" (55c). Además, y este punto es aún más importante, Platón calificó su cosmología como sólo probable: "de modo que acerca de esto conviene que aceptemos el relato probable y no busquemos más allá" (29d). La razón de esa depreciación epistémica es la distancia que existe, dentro del relato cosmogónico que nos narra Platón, entre la naturaleza humana y la divina. Según el Timeo, los humanos fueron creados en un punto relativamente bajo de la escala del ser en comparación con la perfección de los ordenes superiores, por ello Platón afirma que "nosotros dependemos [para conocer] en gran medida de la casualidad y el azar" (24c). Esa imposibilidad de alcanzar una certeza plena, limita la aplicación efectiva de las matemáticas al mundo físico, de tal modo que la propuesta de las series y de los sólidos regulares del Timeo carece de cualquier cálculo concreto que sirva de explicación a los fenómenos.

En contraste con la propuesta del griego, Kepler ya no utiliza los sólidos regulares para fundamentar un atomismo geométrico, sino para desarrollar una arquitectura de los cielos, cuya configuración está confiado en descifrar gracias a la capacidad de la mente humana para comprender con exactitud la matemática.

${ }^{30}$ MC, II $(1992,93)$. La anterior es una cita vía Cicerón de Timeo, 30a.

${ }^{31}$ Timeo, 27a (2006, 170). Literalmente: "sobre la naturaleza de todas partes".

32 "was not necessarily to be taken entirely at its face value" como indica Field $(1998,1)$. 
Esto último se refleja en la forma en que Kepler formuló en el Mysterium su propia versión de una cosmología pitagórica. El astrónomo alemán especuló que Dios trazó los cielos "a la manera de alguno de nuestros arquitectos" (Kepler, $1998,56)$, y que, contrariamente al tópico de que el arte imita a la naturaleza, la naturaleza fue diseñada atendiendo "al modo de construir del hombre que había de venir después". La metáfora del arquitecto tiene una doble vertiente que explota Kepler en su discurso. Por una parte, eleva la matemática a la cima de la cosmología y, con esa ascensión, asume la competencia de los astrónomos en temas filosóficos y cosmológicos hasta entonces vedados a los matemáticos; ${ }^{33} \mathrm{al}$ mismo tiempo se establece un modelo computable para investigar las distancias planetarias e intentar cuantificar la fuerza del Sol que causa los movimientos. Como complemento necesario de los dos puntos anteriores, Kepler afirma la existencia de una simetría entre la razón divina del cosmos y la mente humana, pues cree que Dios infundió en la mente los mismos arquetipos con que dispuso y construyó los cielos; como escribe en su posterior obra De Stella Nova (1606):

Por lo tanto, queriendo persuadir [Dios] a los hombres sobre la astronomía, no usó palabras efímeras, sino que expresó esta voz en la Naturaleza y medida de los cuerpos celestes, los cuales participan de la Geometría junto con la mente humana, y en la disposición de las sombras de la Luna y la Tierra. $^{34}$

Desde esa convicción del poder de la razón humana para comprender matemáticamente el cosmos, Kepler se plantea tres temas fundamentales: la razón

\footnotetext{
${ }^{33}$ La elevación de la matemática al rango de filosofía y la filosofía natural al de teología es una constante de los pensadores renacentistas de filiación pitagórica. El propio Copérnico expresó en las primeras secciones del De Revolutionibus su convicción de que la matemática y la filosofía se deben practicar juntas y que la astronomía es la cabeza de las matemáticas. Véase: Copérnico, Sobre las revoluciones de los orbes celestes, p. 13. Esas ideas aparecen en el prefacio original que fue sustituido en las ediciones de 1543 y 1566 por la Carta a Pablo III, y sólo se recupera en el siglo XIX a partir del manuscrito. Al respecto, la nueva edición del De revolutionibus de de Lerner-Segonds-Verdet (2015, III: 585-594).

34 "Sic persuadere volens hominibus astronomiam, non usus est verbis transeuntibus; sed expressit hanc vocem in Natura et commensuratione coelestium, Geometria participantium cum humana mente, et institutione umbrarum Lunae et Telluris." Kepler, De Stella Nova, XXX $(K G W, \mathrm{I}, 338)$.
} 
de la división del cielo, sus dimensiones y la velocidad de los planetas: "Tres cosas había en concreto sobre las cuales yo insistentemente quería saber por qué eran así y no de otra manera: el número, la magnitud y el movimiento de los orbes (Numerus, Quantitas et Motus Orbium)". ${ }^{35} \mathrm{Si}$ el diseño del cosmos era racionalmente armónico, entonces debía existir un principio de razón suficiente para todas las relaciones en los cielos. En tal sentido Kepler confiaba en poder justificar el hecho de que en la teoría copernicana sólo existieran seis planetas, la proporción de sus distancias mutuas y también de sus velocidades. Kepler afirmó que se atrevió [auderem] a preguntar esto debido a "la maravillosa armonía de las cosas inmóviles, el Sol, las estrellas fijas y el espacio intermedio”. ${ }^{36}$ Para el astrónomo alemán la perfección de las tres regiones inmóviles celestes copernicanas es tan manifiesta que no teme compararlas con la Trinidad cristiana y además en extenderla hacia los objetos móviles.

El realismo matemático de Kepler y su copernicanismo son dos cuestiones que no se desarrollan de forma independiente, sino que se refuerzan mutuamente. Aunque es cierto que Kepler conserva en el Mysterium bases cosmológicas tradicionales, como la idea de que las trayectorias planetarias eran perfectamente circulares y su velocidad angular uniforme, también es verdad que el germano reclamaba una correlación entre las teorías y los fenómenos que fuera más allá de la predicción de las posiciones planetarias. Para Kepler el lugar del Sol y de los planetas, así como sus velocidades, debían dar cuenta de sus interacciones físicas. En tal sentido el geocentrismo y el heliocentrismo, aunque matemáticamente equivalentes, no eran físicamente intercambiables.

\section{Precisión matemática con bases teológicas}

Para explicar la perfecta correlación entre el mundo físico y las matemáticas, Kepler compuso una cosmogonía que parte de la metáfora (platónica) del arquitecto en conjunción con el Génesis bíblico. Kepler afirma:

${ }^{35} M C$, "Praefatio ad lectorem" (1992, 66; $\left.K G W, \mathrm{I}: 9\right)$.

36 "Et tria potissimum erant, quorum ego causas, cur ita, non aliter essent, pertinaciter quaerebam, Numerus, Quantitas, et Motus Orbium.” $K G W$, I: 9. 
La cantidad [quantitas], efectivamente, fue creada al principio junto con la materia y el cielo el segundo día. Pero (pensaba yo), si según la cantidad y proporción de los seis cielos establecidos por Copérnico, tan sólo se pudiesen hallar cinco figuras, de entre las infinitas posibles, que tuviesen propiedades particulares sobre las demás, el asunto quedaría resuelto a satisfacción. ${ }^{37}$

Y páginas más delante concluye:

Al comienzo Dios creó la materia [corpus]; y si conocemos su definición creo que resultará meridianamente claro por qué al principio Dios creó la materia y no otra cosa. Digo que la cantidad fue el propósito de Dios, y para su obtención fue necesario recurrir a todas las cosas que pertenecen a la esencia de la materia, de tal modo que la cantidad de la materia, en tanto que materia, viene a ser como la forma [quaedam forma] y el origen de la definición [...] es mucho más verosímil que [...] las cantidades existieron para que estas cosas se dieran en la realidad y que, para que la cantidad se diera en la realidad, la materia fue creada al principio de todo. ${ }^{38}$

De los tres principales presupuestos de esta cosmología, que son(1) la equivalencia entre geometría y los cuerpos materiales usando la ambigüedad inherente al concepto de quantitas; (2) los cuerpos se convierten en una especie de forma o vehículo de expresión de las cantidades geométricas; y (3) la deducción de propiedades y normas del cielo es posible a-priori desde la matemática. ${ }^{39} \mathrm{Se}$ desprende que los cuerpos físicos están determinados por el orden geométrico.

\footnotetext{
${ }^{37} M C$, "Praefatio ad lectorem" (1992, 69 y 70; KGW, I: 13).

${ }^{38} M C$, II $(1992,92-94)$. Aunque en la segunda edición Kepler matizó esto, argumentando que las ideas en realidad son coeternas con la mente divina, la precesión temporal del acto creador sirve para justificar el uso de la geometría por el hombre como paradigma de los cuerpos físicos. Esto llevó a Kepler a postular la exactitud entre el cálculo y la observación como indispensable para convalidar la verdad de una hipótesis astronómica.

${ }^{39}$ Esta idea Kepler la expresa con toda claridad en el cap. XI, donde escribió que "deduje propiedades naturales de los planetas de cosas inmateriales y de figuras matemáticas (quòd naturales planetarum proprietates ex rebus immaterialibus et figuris mathematicis deduxi)" $M C, \mathrm{XI}(\mathrm{KGW}, \mathrm{I}: 37 ; 1992,123)$.
} 
La cosmogonía de Kepler implicaba un perfecto empate entre los cálculos teóricos y los datos conocidos de las distancias planetarias. La exactitud se convirtió así en un criterio de demostración de la realidad de una teoría así planteada. El propio Kepler tiene conciencia del cambio radical que está proponiendo y, de hecho, rechaza que sus ideas fueran hipótesis astronómicas sino que las tiene por tesis cosmológicas. En este sentido es importante señalar lo que se entendía por hipótesis en el siglo XVI, como hizo Owen Gingerich:

Rheticus, in common with most other sixteenth-century astronomical writers, uses "hypothesis" to mean an arbitrary geometrical device by which the observed celestial motions can be explained. Included within this set of geometrical devices was the grand hypothesis of them all, the heliocentric concept itself. The ultimate nature of the hypotheses, that is to say, whether they were hypothetical models or something real became a fundamental issue in deciding on the relevance of the heliocentric idea. $(1993,286)^{40}$

En absoluto contraste con la idea común de hacer astronomía, Kepler aceptó el reto lanzado por Petrus Ramus a mediados del siglo XVI de hacer una astronomía "sin hipótesis". En otras palabras, el germano busca una astronomía construida sin artificios geométricos, hilando únicamente argumentos físicos y demostraciones matemáticas. El primer gran paso de esa nueva astronomía fue restituir al Sol como centro absoluto del sínodo planetario, punto en que ni siquiera el propio Copérnico lo había colocado en esa posición.

Furthermore, Kepler recognized that although in the Copernican system the sun was near the center, it played no physical role. Kepler argued that the sun's centrality was crucial, for the sun itself must provide the driving

40 "Rhéticus, al igual que la mayoría de los escritores astronómicos del siglo XVI, utiliza "hipótesis" para referirse a un artificio geométrico arbitrario por el cual se pueden explicar los movimientos celestes observados. Dentro de este conjunto de artificios geométricos estaba incluida la gran hipótesis de todas ellas, el concepto heliocéntrico mismo. La naturaleza última de las hipótesis, es decir, si eran modelos hipotéticos o algo real, se convirtió en un tema fundamental para decidir la relevancia de la idea heliocéntrica”. 
force to keep the planets in motion, and he set out for the fist time to show this connection mathematically. (Gingerich, 1993, 286) ${ }^{41}$

Este alto estándar de precisión resultó en la práctica muy difícil de alcanzar. Al comenzar a trabajar con las distancias planetarias Kepler se enfrentó a tres grandes problemas: la fiabilidad de las observaciones, su propia falta de destreza en las técnicas matemáticas y las ambigüedades que existían en los modelos matemáticos. El primero de estos problemas se hizo notar casi de inmediato, pues al comenzar a redactar el Mysterium, Kepler confiaba en los datos de Copérnico tal y como se los había transmitido su maestro Mästlin en Tübingen. Sin embargo, al calcular las proporciones entre planetas según la teoría de los sólidos, las discrepancias entre los datos usados y los resultados de los cálculos dejaban mucho que desear. Como hace notar Kepler en el cap. XVIII del Mysterium:

En efecto, ninguno de los planetas conserva el arco que le fue atribuido por Copérnico. A Saturno le quité 41'; a Júpiter, 6'; a Marte le añadí 30'; a Venus le aumenté la enormidad de $2^{\circ} 18^{\prime}$; y a Mercurio, 61'. Por tanto, quienes desean examinar las cosas con exactitud [exactius] considerarán que, puesto que el cálculo a partir de los sólidos no concuerda exactamente con los datos de Copérnico y con sus valores, todo mi trabajo es un puro juego. Y salvo que responda a esto habré perdido la causa en virtud de mi propia sentencia. ${ }^{42}$

Aunque a párrafo seguido se esfuerza por restarle importancia al problema, lo cierto es que Kepler mismo no se sentía a gusto con las discrepancias. Los perturbadores cambios en las distancias relativas que no eran explicados por los modelos astronómicos atormentaban a Kepler y éste le confió sus dudas a su

\footnotetext{
41 "Además, Kepler reconoció que aunque en el sistema copernicano el sol estaba cerca del centro, no desempeñaba ningún papel físico. Kepler argumentó que la centralidad del sol era crucial, ya que el sol mismo debía proporcionar la fuerza motriz para mantener en movimiento a los planetas, y se propuso por primera vez mostrar esta conexión matemáticamente". ${ }^{42} M C$, XVIII $(1992,175)$.
} 
maestro Mästlin confesando su "astronomiae Copenicanae scientiae inopiam", 43 y preguntándole hasta qué punto eran fiables las observaciones consignadas por Copérnico y si podía usarlas en su libro.

Kepler admite también que no sólo le preocupaba la precisión de los datos, sino el funcionamiento de los modelos astronómicos: "Dado que estas dificultades [sobre las excentricidades] y otras del mismo género me perturbasen no poco y anduviese necesitado de consejo, como quien no sabe recomponer las ruedas dispersas de un autómata, Maestlin me consoló y me exhortó a prescindir de tan minuciosa precisión". "44 La consulta de Kepler a su maestro no se limitó a preguntar sólo sobre la exactitud de las observaciones, pues como señala arriba, sus dudas se ampliaban hasta la teoría planetaria en general. Como explica Anthony Grafton: "In fact, he [Kepler] asked a number of questions about the proper determination of planetary distances, the value of various observations and sets of observations, and the possibility of adjusting the empirical data to make it fit his hypothesis". ${ }^{45}$ Los interrogantes de Kepler dan muestra además de su falta de dominio en la astronomía práctica; en consecuencia la respuesta de Mästlin no se limitó a señalar hasta que punto los datos de Copérnico eran deficientes ni a recomendar sólo el uso de las Tablas Pruténicas de Erasmo Reinhold, sino que también terminó por componer un pequeño e importante tratado de teoría planetaria que apareció como apéndice del Mysterium, el De dimensionibus orbium et sphaerarum coelestium. ${ }^{46}$

Pese al esfuerzo de Mästlin y del propio Kepler por trabajar con los datos disponibles, la obtención de distancias confiables era de suma importancia tanto para la propuesta de los sólidos regulares como para perfeccionar la teoría de la fuerza solar. En ambos casos no se podía llegar a resultados concluyentes sin datos fiables. El propio planteamiento de Kepler, que hermanaba física y matemática, requiere que incluso las demostraciones a priori de los fenómenos fueran comprobadas a posteriori para verificar su validez. Así el paso lógico en la carrera de Kepler era obtener un conjunto de observaciones más fiables, así como también ampliar su dominio de las técnicas matemáticas disponibles para abordar los

\footnotetext{
${ }^{43}$ Carta de Kepler a Mästlin del 3 de octubre de 1595. KGW, XIII, Nr. 23, 36.

${ }^{44}$ MC, XVIII $(1992,180)$.

${ }^{45}$ Grafton, 1973, 524 "De hecho, [Kepler] formuló una serie de preguntas sobre la correcta determinación de las distancias planetarias, sobre el valor de varias observaciones y conjuntos de observaciones y sobre la posibilidad de ajustar los datos empíricos para adaptarlos a su hipótesis".

${ }^{46} K G W \mathrm{I}, 132-145$.
} 
problemas abiertos desde nuevas perspectivas según las necesidades de cada caso. En tal sentido el acercamiento a Tycho Brahe y su "tesoro de observaciones" no es un giro en la trayectoria de Kepler, sino una ampliación del programa planteado en el Mysterium.

Aún con sus carencias el libro de Kepler es un ingenioso despliegue de temas que seguirán presentes a lo largo de sus obras más maduras. Después de dedicar la mayor parte del Mysterium casi exclusivamente a los sólidos, hacia el final del libro el germano retoma su teoría de la fuerza solar y trata de establecer un modelo matemático para calcularla. El procedimiento, aunque erróneo en su resultado, nos muestra el camino de construcción y acercamiento que Kepler empleaba para sus teorías físicas. La teoría solar de Kepler aparece enunciada en el cap. XX del Mysterium de la siguiente manera:

Si no obstante quisiéramos aproximarnos aún más a la verdad y tener expectativas respecto a alguna igualdad de las proporciones, sería preciso establecer una de dos: que las almas motrices son más débiles cuanto más se hallan lejos del Sol, o bien que sólo hay un alma motriz en el centro de todos los orbes, esto es en el Sol, que empuja más fuertemente a un cuerpo cuanto más próximo se halla, mientras que para los más lejanos, debido a la distancia y al debilitamiento de su fuerza, como si languideciera. Pues tal y como ocurre con la fuente de luz en el Sol, y el origen del círculo está en el lugar del Sol, esto es, en el centro, así aquí la vida, el movimiento y el alma del mundo reside en el mismo Sol, para que así ocurra que el reposo es para las fijas, para los planetas los actos segundos del movimiento y para el Sol el propio acto primero, que es incomparablemente más noble que los actos segundos en todas las cosas. $(1994,193)$

Esta aproximación comienza por descartar la posibilidad de múltiples almas motrices, una en cada planeta, que establecerían criterios propios para cada velocidad, optando por una única alma residente en el centro físico del sistema que sería el cuerpo solar. Dicha elección es congruente con la progresión descendente de velocidades y distancias de cada planeta como se establece en el heliocentrismo. Establecido ese planteamiento inicial, se busca una relación directa entre los periodos y el tamaño de las trayectorias de cada par sucesivo de 
planetas que muestre que ambos están sujetos a la misma fuerza, suponiendo que ésta sea idéntica en magnitud. Kepler construye una sencilla igualdad para contrastarla con los datos bien conocidos de los periodos orbitales: La razón entre periodos de planetas sucesivos es igual a la razón de sus radios o P1:P2::r1:r2, usando la distancia media para el radio. En otras palabras, el germano explora la posibilidad de que el incremento directo de sus radios sea proporcional al tiempo de revolución: "Lo que significa que, por ejemplo, la proporción de los 88 días de Mercurio a los 225 de Venus habría de ser la misma que el radio del orbe de Mercurio al radio del orbe de Venus". ${ }^{47}$ La evidente desproporción entre ambas cosas confirma que existe algún otro factor que interviene en el movimiento, Kepler pensaba así que la fuerza solar se debilita de forma análoga a la luz. Establecido esto continúa escribiendo:

Pero ahora se añade a esta proporción de los movimientos el debilitamiento del alma motriz para el más lejano. Por lo tanto supongamos, y es cosa bien plausible, que el Sol proporciona movimiento en la misma razón en que proporciona luz. Pero la proporción en que la luz se propaga desde el centro, la dan los ópticos. Pues cuanto más luz hay en un círculo pequeño y más atenuada en el grande, la medida de esta atenuación habrá de buscarse mediante la propia relación de los círculos, y esto tanto en el caso de la luz como de la fuerza motriz [...] Luego de aquí se sigue que por cada una elongación respecto al Sol se produce un doble aumento periódico; y al contrario, un incremento periódico es doble respecto a la diferencia de "distancias". $(1994,194)^{48}$

El ajuste por el decremento llevó a una nueva formulación, que consiste en sumar la mitad de la diferencia entre los periodos al periodo menor y comparar la proporción entre esta suma con la proporción entre radios: P1 + 1/2(P2-P1):P1 :: r1:r2. El avance es casi a ciegas, Kepler utiliza la cuarta proporcional y va ajustando elementos para que se aproximen a los resultados conocidos. En la segunda edición (en la octava nota al cap. XX), Kepler mismo nos aclara que "[entonces] caminaba con pasos inseguros y palabras imprecisas, y no con una

${ }^{47} M C, \mathrm{XX}(1994,194)$.

${ }^{48} \mathrm{Ibid}$. 
ley aritmética" ${ }^{49}$ Las herramientas matemáticas que se muestran en el Mysterium se basan casi por completo en Euclides y muestran un nivel de dominio no muy sólido. Por contraste, las profundas intuiciones físicas impelían a usar incluso las técnicas básicas que poseía Kepler con creatividad. El propio Eloy Rada afirma que "como él [Kepler] reconoce en la nota [esta fórmula] era un espejismo [...] en cambio aparece la idea de fraccionar magnitudes a comparar entre 'unidades' correspondientes tan pequeñas como se quiera imaginar (aunque en este caso no se imaginen muy pequeñas de modo explícito)" ${ }^{50}$ Aún con sus limitaciones, la búsqueda de exactitud impulsaba al germano a explorar los límites de la propia matemática para comprender el funcionamiento del mundo físico.

\section{Conclusiones}

La especulación de la fuerza física del Sol y su relación con los periodos de los planetas, que a nosotros nos parece como uno de los puntos más importantes del Mysterium, fue la sección que más rechazo suscitó, mientras que la especulación geométricas de los sólidos fue mejor recibida por su parecido a las investigación armónicas de la astrología. Para los astrónomos de la época la construcción de una física astronómica era un proyecto muy difícil de aceptar:

However, there was one point to which astronomers reacted uniformly negatively: they all agreed that Kepler's attempt to account for the function of the equant on the basis of his planet-moving force was ill-conceived. They considered it inappropriate -even dangerous- to apply physical reasoning to mathematical planetary theory. (Voelkel, 2001, 5) (1 $^{5}$

Incluso en el mismo Mästlin, principal promotor de una astronomía matemática a priori, le escribió a Kepler advirtiéndole que adentrarse demasiado en

\footnotetext{
${ }^{49} M C, \mathrm{XX}(1994,197)$.

${ }^{50}$ Nota 6 al cap. XX del $M C(1994,249)$.

51 "Sin embargo, hubo un punto en el que los astrónomos reaccionaron uniformemente negativamente: todos estaban de acuerdo en que el intento de Kepler de explicar la función del ecuante sobre la base de su fuerza de movimiento planetario estaba mal concebido. Consideraron inapropiado -aun peligroso- aplicar el razonamiento físico a la teoría matemática planetaria”.
} 
consideraciones físicas podría traer como resultado "ruinam totis Astronomiae". 52 Es decir, era perfectamente válido armar una especulación geométrica pero existía un fuerte rechazo a permitir que un astrónomo entrara en los terrenos de la física e, institucionalmente, incluso una oposición aún mayor a que invadiera el ámbito de la teología. Sin embargo, para Kepler las tres cosas eran diferentes aspectos de un mismo problema, su gran armonía no sólo precisaba vincular la distancia de los planetas al Sol con sus periodos, también quería ofrecer la razón del número de planetas, su patrón de distribución en el espacio e incluso explicar porque existía tal correlación entre matemáticas y física o demostrar que el heliocentrismo y las Sagradas Escrituras eran esencial lo mismo según su neoplatónica y protestante interpretación. La integración, no sólo congregación, de todos estos aspectos es lo que constituye el estrato profundo del pensamiento científico kepleriano, su fuerza especulativa y su creatividad. Todos estos postulados centrales de su obra juvenil se convirtieron en su programa de investigación de toda su carrera, y aunque ya en su obra tardía escribió que la Astronomía debía cimentarse en ciencias superiores, ${ }^{53}$ esto no implicaba regresar a un estado de tutela por parte de los filósofos o teólogos, sino el deber de adentrarse en esos terrenos por parte de los astrónomos.

\section{Agradecimientos}

Agradezco al CONACYT de México por financiar mi investigación. Así también como a Miguel Ángel Granada por su infinito apoyo y a Patrick Boner por sus siempre pacientes y oportunos consejos.

${ }_{52}$ Carta de Mästlin a Kepler del 9 de marzo de 1597, KGW, XIII, Nr. 63, 111.

53 Westman, 1972, 261. 


\section{Bibliografía}

Blair, Ann (1990). "Tycho Brahe's critique of Copernicus and the Copernican system", Journal of the History of Ideas 51 (3), pp. 355-377.

Copérnico, Nicolás (2015). De revolutionibus orbium coelestium/ Des révolutions des orbes célestes, traductores: Michel-Pierre Lerner, Alain Philippe Segonds y Jean-Pierre Verdet, 3 vol., París: Les Belles Lettres.

-(1989). Sobre las revoluciones de los orbes celestes, editor: Carlos Mingues, Madrid: Tecnos.

Field, J. V. (1998). Kepler's geometrical cosmology, Chicago: University of Chicago Press.

Gingerich, Owen (1993). The eye of heaven: Ptolemy, Copernicus, Kepler, New York: American Institute of Physics.

Goldstein, Bernard; Hon, G. (2005). "Kepler's Move from Orbs to Orbits: Documenting a Revolutionary Scientific Concept", Perspectives on Science, vol. 13, no. 1, pp. 74-111.

Grafton, Anthony (1973). "Maestlin on Copernicus" en Proceedings of the American Philosophical Society, 117, 6, pp. 523-550.

Granada, M. A.; Boner, P.; Tesscini, D. (2016). Unifying heaven and earth: essays in the history of early modern cosmology. Barcelona: Edicions de la Universitat de Barcelona D.L.

GranadA, Miguel Ángel (2010). “'A quo moventur planetae?’ Kepler et la question de l'agent du mouvement planétaire après la disparition des orbes solides”, Galilaeana. Journal of Galilean Studies, 7, pp. 111-141.

Grasshoff, Gerd (2012). "Michael Maestlin's Mystery: Theory Building with Diagrams", Journal for the History of Astronomy, Vol. 43, No. 1, pp. 57-74.

Heller, Herd (1996). "Copemican Ideas in Sixteenth Century France”, Renaissance and Reformation, XX, 1, pp. 5-26.

Jardine, Nicholas (1984). The Birth of History and Philosophy of Science. Kepler's "A Defence of Tycho against Ursus" with Essays on its Provenance and Significance, Cambridge: Cambridge University Press.

Kepler, Johannes (1930-2009). Gesammelte Werke, editor: Max Caspar et al., 20 vol., Munich: C. H. Beck.

-(1992). El secreto del universo, trad. Eloy Rada García, Madrid: Alianza.

Koyré, Alexander (1973). The Astronomical Revolution, London: Mathuen.

Methuen, Charlotte (1998). Kepler's Tübingen. Stimulus to a Theological Mathematics, Aldershot: Ashgate. 
Noirot, Caroline; Ordine, Nuccio (2012). Omnia in uno. Hommage à Alain-Philippe Segonds, París: Les Belles Lettres.

Platón (2006). Diálogos VI, traducción: Francisco Lisi, Madrid: Gredos.

Proclo (1970). A Commentary on the First Book of Euclid's Elements, traducción: Glenn R. Morrow, Princeton: Princeton University Press.

Stephenson, Bruce (1987). Kepler's physical cosmology, New York: Springer-Verlag.

VAn Helden, Albert (1985). Measuring the universe: cosmic dimensions from Aristarchus to Halley. Chicago: University of Chicago Press.

Voelkel, R. James (2001). The composition of Kepler's Astronomia Nova, Princeton: Princeton University Press.

-(1999). Johannes Kepler and the New Astronomy, Nueva York/Oxford: Oxford University Press.

Westman, Robert S. (1975). "The Melanchthon circle: Rheticus and the Wittenberg interpretation of the copernican theory", Isis 66, pp. 165-193.

-(1972). "Kepler's theory of hypothesis and the 'realist dilemma' ", Studies in history and philosophy of science 3, pp. 233-264

Recibido : 15/09/2016

Aceptado : 27/01/2017

ENDOXA está bajo una licencia de Creative Commons Reconocimiento-NoComercial-SinObraDerivada 4.0 Internacional 
